\title{
Molecular Cloning of the Canine Fragile Histidine Triad (FHIT) Gene and Fhit Protein Expression in Canine Peripheral Blood Mononuclear Cells
}

\author{
Hiroko HIRAOKA ${ }^{1)}$, Koji MINAMI ${ }^{1)}$, Naoki KANEKO ${ }^{1)}$, Takako SHIMOKAWA MIYAMA ${ }^{1)}$, Takuya MIZUNO ${ }^{1)}$ and \\ Masaru OKUDA ${ }^{1) *}$ \\ ${ }^{1)}$ Laboratory of Veterinary Internal Medicine, Faculty of Agriculture, Yamaguchi University, 1677-1 Yoshida, Yamaguchi 753-8515, \\ Japan
}

(Received 11 November 2008/Accepted 27 November 2008)

ABSTRACT. A fragile histidine triad (FHIT) gene has been studied as a tumor-associated gene in humans. The aberrant FHIT gene and its protein expression have been reported in many types of human cancers. The present study explored the canine FHIT gene structure and its protein expression in the peripheral blood mononuclear cells of healthy dogs by RT-PCR, RACE and immunoblot analysis. The obtained canine FHIT gene contained nine small exons and was located on canine chromosome 20. Furthermore, we identified an alternative splicing form of the FHIT transcript. The deduced amino acid sequence was well conserved between species, and anti-human Fhit antibody could be used to detect the canine Fhit protein. These findings will be useful for future research. KEY WORDS: antibody, canine, clinical oncology, gene map, molecular identification.

J. Vet. Med. Sci. 71(5): 645-649, 2009

The common fragile sites (CFS) are genomic unstable regions that tend to form gaps and breaks on metaphase chromosomes when cells are exposed to replication impedance, such as by aphidicolin, 5-azacytidine and bromodeoxyuridine [6, 19]. The FRA3B (3p14.2) region is one of the most frequently affected regions in the human genome, in which the instability region extends for 4 megabase pairs (Mbp), and allelic loss or homozygous deletions have been reported in many types of cancer, including lung, renal, esophageal, breast and cervical tumors $[6,7,19]$. Regardless of the unstable region, some genes are located in FRA3B, and in particular, a fragile histidine triad (FHIT) gene has been investigated extensively $[8,19]$. In the human genome, this gene spans $1.5 \mathrm{Mbp}$ within the FRA3B region; however, the final transcript contains only 1.1 kbases coding for a $17 \mathrm{kDa}$ Fhit protein and is composed of 10 exons [14]. Thus, this gene is composed of small exons and extremely large introns.

Aberrant FHIT gene expression and down-regulation of Fhit protein expression have been reported in many types of human tumors. The aberration of the FHIT gene includes some exonic deletions and small sequence insertions in its genome $[2,14,16]$. Moreover, aberrant methylation in the CpG island around exon 1 of the human FHIT gene has been found in numerous cancers, and this aberrant methylation has been shown to be associated with the inactivation of the FHIT gene and decreased expression of the Fhit protein [22, 23, 27]. Decreased expression of the Fhit protein has been found in approximately $50 \%$ to $83 \%$ of human solid tumors, including those of lung, cervical, esophageal and breast cancers, and $40 \%$ to $70 \%$ of hematopoietic malignancies,

\footnotetext{
* CorRespondence to: OKudA, M., Laboratory of Veterinary Internal Medicine, Faculty of Agriculture, Yamaguchi University, Yamaguchi 753-8515, Japan.

e-mail: okudamu@yamaguchi-u.ac.jp
}

including myelocytic leukemia, lymphocytic leukemia and non-Hodgkin's lymphoma [7, 16, 21]. It has also been revealed that FHIT -/- and +/- mice develop spontaneous or $\mathrm{N}$-nitrosomethylbenzylamine (NMBA)-induced tumors [4, 26]. Therefore, the FHIT gene is regarded as a tumor-associated gene. The function of the Fhit protein is considered to be related to modulation of the cell cycle and apoptosis. Some studies have reported that Fhit modulates the mid-S phase DNA checkpoint response through regulation of the checkpoint proteins Hus1 and phosphoChk1 [10, 12]. Other studies have suggested that Fhit suppresses target genes, such as cyclin D1, axin2, Mmp-14 and survivin, by binding directly to $\beta$-catenin [24]. However, the precise function of the Fhit protein has not been clarified.

In dogs, many types of tumor seem to develop in the same manner as in humans. However, the roles of the FHIT gene and Fhit protein have not been explored in canine tumors. Recently, the complete canine genome sequence was clarified by the Whitehead Institute/MIT Center for Genome Research (WICGR) [13] and disclosed in the NCBI genomic databank. However, the canine FHIT gene has yet to be identified, probably because it may be difficult to identify extremely small exons that are surrounded by large introns. In another genomic database, Ensembl, a 661-base fragment of the canine FHIT cDNA sequence has been predicted, but the sequence is incomplete because the open reading frame (ORF) of the FHIT gene does not contain the start codon. Therefore, the present study explored the canine FHIT gene structure. Moreover, a commercially available anti-Fhit antibody was examined for detection of canine Fhit protein expression in order to obtain basic information for future studies concerning the FHIT gene and Fhit protein.

To identify the canine FHIT gene and its structure in the genome, total RNA was extracted from peripheral blood 
mononuclear cells (PBMCs) isolated from a healthy dog using TRI Reagent (Molecular Research Center, Cincinnati, $\mathrm{OH}$, USA), and single-strand cDNA was synthesized using SuperScript $^{\mathrm{TM}}$ II RT (Invitrogen, Carlsbad, CA, U.S.A.) with an oligo-dT primer. The detection primers (FHIT-Fp1 and Rp1: 5'-CAACATCTCATCAAGCCCTC-3' and 5'CATTTCCTCCTCGGATCTCCA-3', respectively) were designed within the predicted coding region based on the human (NCBI_accession No: NM_002012), bovine (NM_001040646), mouse (NM_010210), rat (NM_021774) and canine FHIT gene sequences in the Ensembl database (Ensembl accession No: ENSCAFG00000007194). Using the primers and recombinant TaqDNA polymerase (Invitrogen), RT-PCR was performed with 30 cycles of denaturation $\left(94^{\circ} \mathrm{C}\right.$ for $\left.1 \mathrm{~min}\right)$, annealing $\left(55^{\circ} \mathrm{C}\right.$ for $\left.1 \mathrm{~min}\right)$, and polymerization $\left(72^{\circ} \mathrm{C}\right.$ for $\left.2 \mathrm{~min}\right)$ in the reaction mixture recommended by the manufacture. The resulting PCR product was subjected to direct sequencing using a BigDye Terminator v3.0 Ready Reaction Cycle Sequencing kit (Applied Biosystems, Foster, CA, U.S.A.). A 391-base canine PBMC cDNA was amplified using the F1/Rp1 primers, and direct sequencing of the product revealed that it was well conserved compared to the human and bovine FHIT gene sequences (similarity scores of $89.88 \%$ and $89.49 \%$, respectively). Moreover, the obtained sequence was completely identical to the canine chromosome 20 sequence according to an NCBI database search. Based on this sequence, 5 ' and 3' extension primers were designed (5' and 3' RACE: 5'CGAAGGGGACACACCAGGACATG-3' and 5'TGAAGCATGTTCACGTCACGTTC-3', respectively), and 5' and 3' RACE analyses were performed using a SMART RACE cDNA Amplification kit (Clontech, Mountain View, CA, U.S.A.). The 3' region of the FHIT cDNA was obtained from the polyA sequence by 3' RACE analysis, and it was well conserved among the species examined. In contrast, the 5' non-coding sequence, which corresponded to the human, bovine and mouse exon 1 sequences, could not be obtained by 5' RACE. Therefore, canine exon 1 was presumed from canine chromosome 20 based on the human and bovine exon 1 sequences using BLAST searches, and a sense primer was designed within this region (e1: 5'-TCACTTCCCAGCTGCCAAGATC-3'). In addition, an antisense primer was also designed within the final exon, which was clarified by 3' RACE (eL: 5'-TAAGTACATAGCCCAGGAAGTGTGGAAG-3'). Using these e1/ eL primers, RT-PCR and sequence analysis were performed to obtain $86.6 \%$ of the canine FHIT cDNA sequence. As a result, the 924-base sequence of canine FHIT was obtained, excluding the primer sequences (canine wild-type FHIT transcript; deposited in NCBI_accession No. AB469369). Based on this CDNA sequence for FHIT and the canine chromosome 20 sequence, the canine FHIT gene was predicted to contain nine short exons (30-306 bases) separated by extremely large introns (2,333-494,789 bases, Fig. 1). The human FHIT gene has been shown to contain 10 exons [14]. The difference in the exon numbers between human and canine FHIT resulted from differences in the 5' non-
A

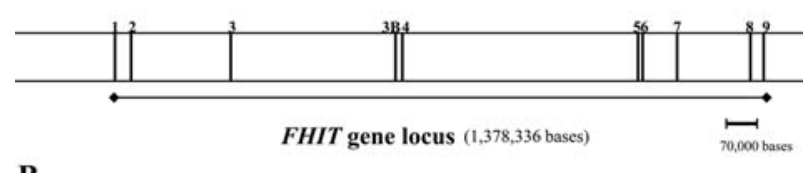

B

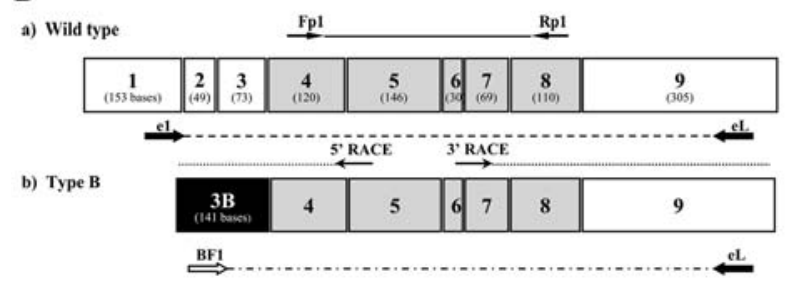

Fig. 1. (A) FHIT gene locus on canine chromosome 20. The FHIT gene exons are surrounded by extremely large introns. Each exon is shown by a black box containing the number of the exon. A specific exon in the type B transcript, exon 3B, is present between exons 3 and 4 of the wild-type transcript. (B) Two types of canine FHIT transcript were obtained. The wildtype transcript consists of nine exons and shows a basically similar structure to the FHIT genes in other species (a). Comparison of the two transcripts shows that the type B transcript includes an exon, exon 3B, in the 5' non-coding region that is not found in the wild-type transcript (b). The open reading frames of these two transcripts (shaded boxes) share the same amino acid sequence. The numbers below the exon numbers indicate the length of each exon, and the arrows indicate the primers used in this study.

coding region, in which the human and canine FHIT genes contain four and three exons, respectively. Exons 1 and 2 of the canine FHIT gene have sequence similarities with those of humans, but exons 3 and 4 of the human FHIT gene do not have any similarity with canine FHIT, which contains only one exon corresponding to the human exon 3 and 4 regions. In contrast, exons 4 to 9 of the canine FHIT gene correspond to exons 5 to 10 of the human FHIT gene, which contain the ORF and 3' non-coding region, and were well conserved between the species. The deduced amino acid sequence of the canine Fhit protein was 149 residues and was shown to have high similarities with those of other species $(98.7 \%, 98.0 \%, 98.0$ and $96.6 \%$ for the rat, mouse, bovine and human, respectively). The human Fhit protein is composed of 147 amino acid residues and represents the second branch of the histidine triad (HIT) protein superfamily, which is characterized by a His-X-His-X-His-XX (X: hydrophobic amino acid) motif [1]. This motif was conserved completely as His-Val-His-Val-His-Val-Leu (a.a. 96-100) in canine Fhit. Amino acids 1-75 in human Fhit, which are required for binding to $\beta$-catenin, were also well conserved in canine Fhit [24]. These results suggest that the canine FHIT gene has presumably similar functions to those in other species.

Interestingly, the 5' non-coding sequence of the cDNA obtained using primers e1/eL was different from that of the cDNA obtained by 5' RACE in the upstream region of exon 4 , which contains the start codon. Hereafter, the former and 
A

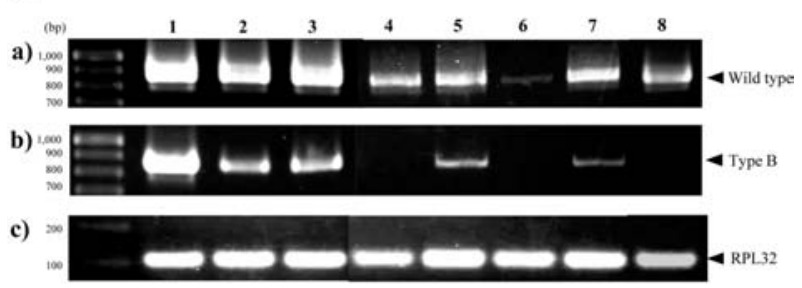

B

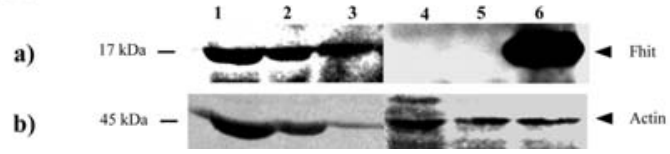

Fig. 2. (A) RT-PCR analysis of the FHIT gene in canine PBMCs and other tissues. The wild-type and type $\mathrm{B}$ transcripts of the FHIT gene were amplified using e1/eL primers (a) and BF1/eL primers (b), respectively. As an internal control, canine ribosomal protein L32 (RPL32), which is a stable housekeeper gene, was used for detection of the canine lymphatic cells and tissues (c) [15]. Lanes 1-3, PBMCs from different dogs; lane 4, lung; lane 5, intestine; lane 6, liver; lane 7, spleen; and lane 8 , thymus. (B) Immunoblotting of the Fhit protein from canine PBMCs and from a canine FHIT gene-transfected cell line. Immunoblot analysis was performed using an anti-human Fhit polyclonal antibody (clone ZR44, Invitrogen) (a). Anti-human actin polyclonal antibody was used for reprobing (b). Lanes 13, PBMCs from different dogs; lane 4, parental KK47 cells; lane 5, empty vector-transfected KK47 cells; and lane 6, canine FHIT-transfected KK47 cells.

later types of the transcripts are defined as 'wild-type' and 'type B', respectively. In a database search, a different 141base sequence was identified between wild-type exons 3 and 4 in canine chromosome 20 and was named exon 3B (Fig. 1B). A sense primer was subsequently designed within exon 3B (BF1: 5'-CTGTGGACAGAAACATCCCACCTG-3'). To confirm expression of the two types of FHIT transcript, RT-PCR was performed using wild-type- and type B-specific primers (e1/eL and BF1/eL, respectively), and cDNAs were obtained from PBMCs, as described above, from the PBMCs of two healthy dogs and from five tissue samples from the lung, intestine, liver, spleen and thymus of another healthy dog. As a result, the wild-type transcript was detected in all eight samples, whereas the type B transcript was identified in the PBMCs, intestine and spleen but not in the lung, liver and thymus (Fig. 2A). Direct sequence analysis of these products confirmed that the wildtype and type B transcripts were only different within the 5' non-coding region, and the type $\mathrm{B}$ transcript had the same sequence after exon 4 as the wild-type transcript (deposited in NCBI_accession No. AB469370). Because the type B transcript was obtained by 5' RACE analysis, it is possible that the 5 ' regions were not synthesized completely during the 5' RACE procedures. However, RT-PCR using the e1/ eL primers amplified only a single band (Fig. 2A). More- over, direct sequence analysis of the product showed that it was the wild-type transcript. These results suggest that the type B transcript was an alternative splicing form of FHIT that shares exons 4 to 9 , but not exon 1 , with the wild-type transcript. In the human FHIT gene, alternative splicing forms have also been reported in normal lymphocytes and other tissues, including the lung, liver, trachea and bronchial epithelium [3, 5, 16, 21, 25]. These transcripts resulted from the loss of entire exons generating splice junctions between the following exons: exons 3 and 6, exons 3 and 9, exons 3 and 8 , exons 4 and 8 or exons 7 and $9[3,16,21]$. More importantly, small sequence insertions previously identified in human lymphocytes were also identified in the canine type B transcript obtained in the present study. In these transcripts, a small 47 or 72 base sequence was inserted between exons 4 and 5, although the sequences of the small insertions have not been reported and could not be determined using their loci in the human genome [16]. However, these small insertions and exon 3B in the canine type B transcript do not seem to influence the ORF of the FHIT genes because the insertions were found in the 5' non-coding region. In the present study, an alternative splicing form of the canine FHIT transcript, type B, was detected in all three PBMC samples and the spleen and intestine. As described above, alternative splicing forms of FHIT transcripts have been reported frequently in human lymphocytes and PBMCs, which contain lymphocytes [16, 21, 25]. This may also apply to the spleen, which expressed the type B transcript in the present study, because the spleen is part of the lymphatic system. Despite belonging to the same system, the thymus of the dog did not express the type B transcript. Moreover, to the best of our knowledge, the alternative splicing forms of FHIT transcripts have not been isolated from the human intestine. The frequency of expression of alternatively splicing forms may differ between tissues, individuals and species. In the present study, the type B transcript was obtained by 5' RACE, and its existence was confirmed by RT-PCR using the BF1 primer (located in exon 3B). Therefore, further studies are necessary to clarify the complete 5' non-coding sequence of the type B transcript as well as its tissue distribution using Northern blot analysis, screening with a cDNA library and immunohistochemistry.

Next, we examined whether a commercially available anti-human Fhit antibody could be used for detection of the canine Fhit protein in immunoblot analysis. The ORF of the canine FHIT gene was introduced into a mammalian expression vector, pcDNA $3.1(+)$ (Invitrogen), and transfected transiently into the human bladder cancer cell line KK47. Briefly, the canine FHIT coding region was amplified by RT-PCR using cDNAs from the PBMCs of the healthy dog with the following primers: 5'-TAGGATCCGCTTCAACCGTGAGGAAATG-3' (TF5) and 5'- ACGATATCTTTC ATGCCTGTAAAGTCA-3' (TF3), which contain restriction enzyme cutting sites for BamHI and EcoRV, respectively. After enzyme treatment, the amplified FHIT gene was ligated into pcDNA3.1(+). The subcloned Fhit expres- 
sion plasmid (pcDNA-Fhit) was used to simultaneously transfect KK47 cells with a plasmid containing a puromycin-resistance gene, pBabePuro, at a molar ratio of 15:1, using Lipofectamine 2000 (Invitrogen). Concurrently, pcDNA3.1(+) was also transfected under the same conditions as an empty vector control (pcDNA). After incubation for $6 \mathrm{hr}$, the cells were washed with PBS, cultured in fresh complete medium for $24 \mathrm{hr}$ and then placed into fresh complete medium. Puromycin $(2.5 \mu \mathrm{g} / \mathrm{m} l)$ was added to the medium $24 \mathrm{hr}$ later. Successfully transfected cells enriched by treatment with puromycin for $24 \mathrm{hr}$ were washed with PBS and harvested. Untransfected KK47 cells, KK47 cells transfected with pcDNA-Fhit, KK47 cells transfected with pcDNA and three PBMC samples isolated from the three above-mentioned healthy dogs were lysed in NP-40 lysis buffer [1\% NP-40, $50 \mathrm{mM}$ Tris (pH 8.0), $150 \mathrm{mM} \mathrm{NaCl}, 1 \%$ SDS, $2 \mu \mathrm{g} / \mathrm{ml}$ leupeptin and $2 \mu \mathrm{g} / \mathrm{ml}$ aprotinin]. The lysates were cleared by centrifugation at 15,000 rpm for $10 \mathrm{~min}$ at $4^{\circ} \mathrm{C}$. Portions of the lysates (10 $\mu$ g for each KK47 sample and $50 \mu$ g for each PBMC sample) were then separated by 15\% SDS-polyacrylamide gel electrophoresis (PAGE) and blotted onto a nitrocellulose membrane. The membrane was incubated in blocking buffer consisting of $5 \%(\mathrm{wt} / \mathrm{vol})$ nonfat dry milk in TBS-T [20 mM Tris-HCl (pH 7.4) and 150 $\mathrm{mM} \mathrm{NaCl}$ containing $0.05 \%$ Tween-20] for $1 \mathrm{hr}$, incubated with the anti-human Fhit antibody (clone ZR44, Invitrogen; diluted 1:500 in TBS-T) for 1-2 $\mathrm{hr}$ at room temperature, rinsed with TBS-T and then incubated with horseradish peroxidase-conjugated goat anti-rabbit IgG (diluted 1:4000 in TBS-T, Invitrogen) for $1 \mathrm{hr}$ at room temperature. After washing with TBS-T, the antibody-antigen complex was visualized using Western Lightning Chemiluminescence Reagent Plus (PerkinElmer) and an LAS-3000 mini chemiluminescence detection system (Fujifilm, Tokyo, Japan). A protein of approximately $17 \mathrm{kDa}$ was identified in all of the canine PBMC samples examined (Fig. 2B). An anti-human Fhit polyclonal antibody was used because it recognizes both human and rat Fhit protein, as described in the manufacturer's data sheet, and the deduced amino acid sequence of the canine Fhit protein obtained in the present study was shown to have high sequence similarity with that of human Fhit protein (96.6\%). Indeed, the size of the approximately $17-\mathrm{kDa}$ protein obtained by immunoblotting was the expected size of canine Fhit. Moreover, the Fhit protein was identified in pcDNA-Fhit-transfected KK47 cells, whereas the Fhit protein was not detected in either the untransfected KK47 cells or the empty vector transfected KK47 cells (Fig. 2B), confirming that the anti-human Fhit antibody used in the present study reacted with canine Fhit.

Although two types of canine FHIT transcript were identified from the PBMCs of the healthy dogs, no other size variants of Fhit protein were observed. This was not inconsistent with our prediction because the type B transcript also contains the same ORF as the wild-type transcript. Thus, the type B transcript may have an additive effect on Fhit protein expression and coexist with the wild-type transcript in normal canine lymphocytes. In humans, it has also been reported that alternative transcripts coexist with wild-type transcripts in lymphocytes, and only a single Fhit protein has been detected [16]. The wild-type FHIT transcript in human tumor cells has been reported to be silenced by CpGisland methylation in the upstream region of exon 1 [22]. Therefore, the alternative splicing form of the FHIT transcript including the type B transcript obtained in the present study may play a role in an alternative Fhit-expressing system. Further studies are necessary to clarify this point.

This study clarified the canine FHIT gene structure and its protein expression pattern. The basic form of the canine FHIT transcript had been shown to have small exons separated by very large introns, and this unique structure is basically conserved in human, bovine, rat and mouse FHIT genes. Moreover, its amino acid sequence was extremely similar to those of the other species. In the human and mouse, the FHIT gene is located in the unstable fragile regions of the genomes, FRA3B and Fra14A2, respectively $[18,19]$. A previous study reported the canine folate/thymidine depleted and aphidicolin-inducible fragile site using PBMCs from the Boxer and Doberman Pinscher breeds. The chromosomal fragile sites varied depending on breed and age, but chromosome 20, which contains the canine FHIT gene locus, was not identified as a fragile site [20]. However, the FRA3B/Fra14A2 loci are conserved in ATrich sequences with numerous short and long repeats throughout the region $[9,18]$. The intronic regions of the canine FHIT gene also have AT-rich short repeat sequences (data not shown). This observation suggests that the canine FHIT gene is also located in a fragile chromosomal site. Furthermore, the present study demonstrated that the antihuman Fhit antibody can be used for study of canine Fhit protein expression. In humans, more than 700 papers have been published concerning FHIT gene expression and Fhit protein function because loss of Fhit protein has been observed in many types of human cancer [7]. Recently, an FHIT gene replacement therapy using an adenoviral vector has also been studied at a preclinical stage [11, 17]. The basic information about the canine FHIT gene and Fhit protein obtained in this study will be useful in clarifying the relationship between FHIT gene expression and cancer development in dogs.

This research was supported by a grant-in-aid from the Ministry of Education, Culture, Sports, Science and Technology of Japan (19390416). The authors would like to acknowledge the technical expertise of the DNA Core Facility of the Center for Gene Research, Yamaguchi University.

\section{REFERENCES}

1. Brenner, C. 2002. Hint, Fhit, and GalT: function, structure, evolution, and mechanism of three branches of the histidine triad superfamily of nucleotide hydrolases and transferases. Biochemistry 41: 9003-9014.

2. Druck, T., Hadaczek, P., Fu, T. B., Ohata, M., Siprashvili, Z., Baffa, R., Negrini, M., Kastury, K., Veronese, M. L., Rosen, D., Rothstein, J., McCue, P., Cotticelli, M. G., Inoue, H., Croce, C. M. and Huebner, K. 1997. Structure and expression 
of the human FHIT gene in normal and tumor cells. Cancer Res. 57: 504-512.

3. Fong, K. M., Biesterveld, E. J., Virmani, A., Wistuba, I., Sekido, Y., Bader, S. A., Ahmadian, M., Ong, S. T., Rassool, F. V., Zimmerman, P. V., Giaccone, G., Gazdar, A. F. and Minna, J. D. 1997. FHIT and FRA3B 3p14.2 allele loss are common in lung cancer and preneoplastic bronchial lesions and are associated with cancer-related FHIT cDNA splicing aberrations. Cancer Res. 57: 2256-2267.

4. Fong, L. Y., Fidanza, V., Zanesi, N., Lock, L. F., Siracusa, L. D., Mancini, R., Siprashvili, Z., Ottey, M., Martin, S. E., Druck, T., McCue, P. A., Croce, C. M. and Huebner, K. 2000. Muir-Torre-like syndrome in Fhit-deficient mice. Proc. Natl. Acad. Sci. U.S.A. 97: 4742-4747.

5. Gayther, S. A., Barski, P., Batley, S., Li, L., de Foy, K. A., Cohen, S. N., Ponder, B. A. and Caldas, C. 1997. Aberrant splicing of the TSG101 and FHIT genes occurs frequently in multiple malignancies and in normal tissues and mimics alterations previously described in tumors. Oncogene 15: 21192126.

6. Glover, T. W. 2006. Common fragile sites. Cancer Lett. 232: 4-12.

7. Huebner, K. and Croce, C. M. 2003. Cancer and the FRA3B/ FHIT fragile locus: it’s a HIT. Br. J. Cancer 88: 1501-1506.

8. Iliopoulos, D., Guler, G., Han, S.Y., Druck, T., Ottey, M., McCorkell, K. A. and Huebner, K. 2006. Roles of FHIT and WWOX fragile genes in cancer. Cancer Lett. 232: 27-36.

9. Inoue, H., Ishii, H., Alder, H., Snyder, E., Druck, T., Huebner, K. and Croce, C. M. 1997. Sequence of the FRA3B common fragile region: implications for the mechanism of FHIT deletion. Proc. Natl. Acad. Sci. U.S.A. 94: 14584-14589.

10. Ishii, H., Wang, Y. and Huebner, K. 2007. A Fhit-ing role in the DNA checkpoint response. Cell Cycle 6: 1044-1048.

11. Ishii, H., Dumon, K. R. Vacchione, A., Fong, L. Y., Baffa, R., Huebner, K. and Croce, C. M. 2001. Potential cancer therapy with the fragile histidine triad gene. JAMA 286: 2441-2449.

12. Ishii, H., Mimori, K., Inoue, H., Inageta, T., Ishikawa, K., Semba, S., Druck, 20, Trapasso, F., Tani, K., Vecchione, A., Croce, C. M., Mori, M. and Huebner, K. 2006. Fhit modulates the DNA damage checkpoint response. Cancer Res. 66: 11287-11292.

13. Lindblad-Toh K, Wade, C. M., Mikkelsen, T. S., et al. 2005. Genome sequence, comparative analysis and haplotype structure of the domestic dog. Nature 438: 803-819.

14. Ohata, M., Inoue, H., Cotticelli, M. G., Kastury, K., Baffa, R., Palazzo, J., Siprashvili, Z., Mori, M., McCue, P., Druck, T., Corce, C. M. and Huebner, K. 1996. The FHIT gene, spanning the chromosome 3p14.2 fragile site and renal carcinoma-associated $t(3 ; 8)$ breakpoint, is abnormal in digestive tract cancers. Cell 84: 587-597.

15. Peters, I. R., Peeters, D., Helps, C. R. and Day, M. J. 2007. Development and application of multiple internal reference (housekeeper) gene assays for accurate normalization of canine gene expression studies. Vet. Immunol. Immunopathol. 117: 55-66.

16. Peters, U. R., Hasse, U., Opplinger, E., Tschan, M., Ong, S. T., Rassool, F. V., Borisch, B., Tobler, A. and Fey, M. F. 1999. Aberrant FHIT mRNA transcripts are present in malignant and normal haematopoiesis, but absence of FHIT protein is restricted to leukemia. Oncogene 18: 79-85.

17. Pichiorri, F., Trapasso, F., Palumbo, T., Aqeilan, R. I., Drusco, A., Blaser, B. W., Ilipoulos, D., Caligiuri, M. A., Huebner, K. and Croce, C. M. 2006. Preclinical assessment of FHIT gene replacement therapy in human leukemia using a chimeric adenovirus, Ad5/F35. Clin. Cancer Res. 12: 3494-3501.

18. Shiraishi, T., Druck, T., Mimori, K., Flomenberg, J., Berk, L., Alder, H., Miller, W., Huebner, K. and Croce, C. M. 2001. Sequence conservation at human and mouse orthologous common fragile regions, FRA3B/FHIT and Fra14A2/Fhit. Proc. Natl. Acad. Sci. U.S.A. 98: 5722-5727.

19. Smith, D. I., McAvoy, S., Zhu, Y. and Perez, D. S. 2007. Large common fragile site genes and cancer. Semin. Cancer Biol. 17: 31-41.

20. Stone, D. M., Jackey, P. B. Hancock, D. D. and Prieur, D. J. 1991. Chromosomal fragile site expression in dogs: I breed specific differences. Am. J. Med. Genet. 40: 214-222.

21. Sugimoto, K., Yamada, K., Miyagawa, K., Hirai, H. and Oshimi, K. 1997. Decreased or altered expression of the FHIT gene in human leukemias. Stem Cells 15: 223-228.

22. Tanaka, H., Shimada, Y., Harada, H., Shinoda, M., Hatooka, S., Imamura, M. and Ishizaki, K. 1998. Methylation of the 5' CpG island of the FHIT gene is closely associated with transcriptional inactivation in esophageal squamous cell carcinomas. Cancer Res. 58: 3429-3434.

23. Virmani, A. K., Muller, C., Rathi, A., Zoechbauer-Mueller, S., Mathis, M. and Gazdar, A. F. 2001. Aberrant methylation during cervical carcinogenesis. Clin. Cancer Res. 584: 584-589.

24. Weiske, J., Albring, K. F. and Huber, O. 2007. The tumor suppressor Fhit acts as a repressor of $\beta$-catenin transcriptional activity. Proc. Natl. Acad. Sci. U.S.A. 104: 20344-20349.

25. Yang, H. W., PIAO, H. Y., Taki, T., Chen, T., Hashizume, K., Ohnishi, H., Bessho, F., Yanagisawa, M., Matsuo, Y. and Hayashi, Y. 1999. Pattern of FHIT gene expression in normal and leukemic cells. Int. J. Cancer 81: 897-901.

26. Zanesi, N., Fidanza,V., Fong, L. Y., Mancini, R., Druck, T., Valtieri, M., Rüdiger, T., McCue, P. A., Croce, C. M., Huebner, K. 2001. The tumor spectrum in FHIT-deficient mice. Proc. Natl. Acad. Sci. U.S.A. 98: 10250-10255.

27. Zöchbauer-Müller, S., Fong, K. M., Maitra, A., Lam, S., Gradts, J., Ashfaq, R., Virmani, A. K., Milchgrub, S., Gazdar, A. F. and Minna, J. D. 2001. 5 CpG island methylation of the FHIT gene is correlated with loss of gene expression in lung and breast cancer. Cancer Res. 61: 3581-3585. 